\title{
Unusual presentation of a skin rash
}

\author{
Rajendra Singh, David Teranno
}

Department of Pathology and Laboratory Medicine, The Mount Sinai Hospital and Icahn School of Medicine at Mount Sinai, New York, New York, USA

\section{Correspondence to}

Dr Rajendra Singh, Department of Pathology and Laboratory Medicine, The Mount Sinai Hospital and Icahn School of Medicine, 1 Gustave L. Levy Place, Box-1194, New York, NY 10029, USA;

rajendra.singh@mountsinai.org

Received 6 November 2016 Revised 9 January 2017

Accepted 17 February 2017 Published Online First 17 April 2017

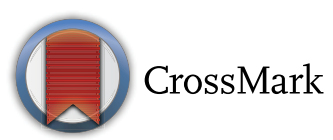

To cite: Singh R, Teranno D. J Clin Pathol 2017;70:1088.

\section{CLINICAL QUESTION}

A 44-year-old man with erythema and oedema of the left abdomen. The duration of the lesion is unknown. Review the high-quality, interactive digital Aperio slide at http://virtualacp.com/JCPCases/ jclinpath-2016-204007/ and consider your diagnosis.
WHAT IS YOUR DIAGNOSIS?
A. Viral xanthem
B. Bacterial folliculitis
C. Secondary syphilis
D. Collagen vascular disease
E. Linear IgA

\section{ANSWER \\ C Secondary syphilis}

\section{DISCUSSION}

The incidence of syphilis has been increasing in the USA since 2001. ${ }^{1}$ The histology of syphilis, though widely studied, still remains a diagnostic challenge, particularly when the clinical presentation does not suggest the diagnosis. We present an overview of the clinical and pathological features commonly associated with cutaneous primary and secondary syphilis, addressing, at the end, the current case.

Treponema pallidum, ${ }^{2}$ as is well known, is the etiological organism of syphilis. Syphilis presents in one of three stages-primary, secondary and tertiary — with primary and secondary syphilis being the most common. Secondary syphilis is most commonly encountered in dermatopathology practice. The anogenital region is the most common location. However, 5\% of cases arise in extragenital sites, which are termed endemic, or non-venereal, syphilis. The oral cavity is the most commonly involved extragenital site, but any site can be involved.

In primary syphilis, the lesion typically appears as a chancre, a punched-out, painless ulcer that develops 20-30 days after exposure to T. pallidum and may be associated with painless lymphadenopathy. These lesions resolve within 1-5 weeks and do not scar.

Secondary syphilis arises 6-8 weeks after the primary chancre. The lesions initially present as $5-10 \mathrm{~mm}$ erythematous macules on the head, face and neck similar to a viral xanthem. A polymorphic papular eruption subsequently occurs and consists of coppery red papules, measuring $3-10 \mathrm{~mm}$ in diameter. Involvement of the palms, soles and oral cavity is highly suspicious for secondary syphilis. However, this classic progression is not always observed and there is clinical significant overlap with other entities and non-specific presentations. Patients can also present with other cutaneous manifestations, some of which overlap with syphilis. These include papulosquamous/psoriasiform lesions, oral ulcers, condylomata, bullous lesions, and erythema muliforme-like folliculitis. Patients with secondary syphilis can also develop fever, headache, weight loss, myalgias and arthralgias. Left untreated, secondary syphilis resolves within 2-10 weeks. After a latent phase, patients can progress to cure with seronegativity; seropositivity without lesions; or develop lesions of tertiary syphilis.

The histopathology of primary and secondary syphilis is non-specific, but there several common findings. In primary syphilis, corresponding to the punched-out appearance of a chancre, histology shows ulceration with adjacent epidermal hyperplasia and lymphohistiocytic and neutrophilic dermal infiltrates. Plasma cells can be present, and in any skin biopsy, should at least raise the suspicion for this disease. Finally, there is often prominent endothelial cell swelling of dermal blood vessels.

The histology of secondary syphilis is much more variable, which accounts for the diagnostic challenge. However, the histology can coincide with the clinical presentation, which can also be normal. For example, a papulosquamous presentation will show psoriasiform changes. The inflammatory reaction patterns include granulomatous inflammation, superficial and deep perivascular and periadnexal lymphocytic inflammation, and a lichenoid-like pattern with or without basal hydropic change. Endothelial cell swelling is common, and a leukocytoclastic vasculitis has been reported, though is extremely rare. Plasma cells tend to be more numerous compared with the lesions of the primary chancre. Neutrophils may be present within the epidermis or stratum corneum, a finding that should also always raise the suspicion for syphilis in any skin biopsy. Keratinocyte necrosis has also been observed. $^{3}$

A recent report reviewed the most common histological features in 106 cases of secondary syphilis. Although interstitial inflammation and endothelial cell swelling were the most common at $91.5 \%$ and $87.7 \%$ of cases, respectively, no single feature was present in $100 \%$ of cases. Furthermore, plasma cells were present in only $69.8 \%$ of cases. Similar to the current case presentation, the study showed that neutrophils were present but in only $32.1 \%$ of cases, which shows their poor sensitivity but higher specificity for syphilis when present. While the findings of this study may represent a sampling issue, it underscores the histological variability of syphilitic lesions. ${ }^{3}$

Definitive diagnosis requires identification of the spirochaete organism, which is now greatly aided by an anti-treponemal immunohistochemical stain. This is a polyclonal antibody against $T$. pallidum surface antigens. The organisms are typically present within the basal layer of the epidermis. The 
reported specificity is nearly $100 \%$ with a sensitivity as high as $90 \% .^{4}$ Interpretation can be challenging because the number of organisms can range from a few to many, and dendrites of melanocytes and Langerhans cells can show artifactual staining. ${ }^{4}$

The current case shows an unusual combination of histological features in a patient who did not have a reported history of immunosuppression or clinical features for syphilis. The

\section{Key messages}

- Incidence of cutaneous secondary syphilis has been increasing in the USA since 2001.

- Treponema pallidum is the etiological organism of syphilis.

- Clinical and histological presentations vary widely. Age (young adult) and unsafe sex practice are associated risk factors.

- Primary syphilis presents clinically as a painless chancre.

- Secondary syphilis presents 6-8 weeks after primary chancre initially as erythematous macules on the head, face, and neck followed by coppery red papules.

- Involvement of palms, soles and the oral cavity is highly suspicious for secondary syphilis.

- No single histological pattern is specific for syphilis, underscored by the current case, which shows features that overlap with viral xanthem, psoriasis, collagen vascular disease and linear $\lg \mathrm{A}$.

- Recent published data indicate that reactive endothelial changes are the most common histological finding, while plasma cells are present in around two-thirds of cases.

- A combination of histological patterns, including reactive endothelial cell changes, psoriasiform hyperplasia, interface changes, neutrophilic infiltrates and plasma cells, should raise suspicion for syphilis.

- Immunohistochemistry for T. pallidum has high specificity (up to $100 \%$ ), but interpretation requires careful examination to avoid false negatives and false positives. biopsy shows a lichenoid and interface dermatitis with significant basal hydropic change and neutrophils. Given the interface changes, collagen vascular disease is a consideration. While the current case show some increase in dermal mucin, periadnexal inflammation was absent and the presence of neutrophils makes it much less likely. The presence of numerous neutrophils within the superficial papillary dermis and focally along the dermoepidermal junction does, however, bring a bullous disorder into the differential diagnosis, including linear IgA. Direct immunofluorescence would exclude this diagnosis once spirochaete infection is excluded. Given that secondary syphilis can present as a maculopapular rash, viral xanthem is also part of the differential diagnosis. However, the lichenoid pattern and the presence of neutrophils argue against this diagnosis. Finally, a folliculitis pattern can be seen clinically, but the current biopsy did not show follicular-centric inflammation or evidence of follicle rupture. While the above differential diagnoses are prudent to consider, one should perform anti-treponemal immunohistochemistry to identify or exclude infection by T. pallidum before undertaking studies such as direct immunofluorescence. In the current case, this was done, and numerous organisms were identified, confirming the diagnosis.

Handling editor Iskander Chaudhry.

Twitter Follow Rajendra Singh @mydermpath

Competing interests None declared.

Provenance and peer review Commissioned; internally peer reviewed.

\section{REFERENCES}

1 CDC (Center for Disease Control and Prevention). 2014 Sexually Transmitted Disease Surveillance: Syphilis. 2015. http://www.cdc.gov/std/stats14/syphilis.htm (accessed 5 Oct 2016).

2 Calonje E, Brenn T, Lazar A, et al. Syphilis. In: McKee's pathology of the skin with clinical correlations. 4th edn. New York, NY: Elsevier Saunders, 2012:465-71 and 808.

3 Flamm A, Parikh K, Xie Q, et al. Histologic features of secondary syphilis: a multicenter retrospective review. J Am Acad Dermatol 2015;73:1025-30.

4 Putri I, Mercer SE, Phelps RG, et al. False-negative anti-treponemal immunohistochemistry in secondary syphilis. Int J Dermatol 2013;52:172-6. 